ISSN 0103-5150

Fisioter. Mov., Curitiba, v. 26, n. 3, p. 639-645, jul./set. 2013

Licenciado sob uma Licença Creative Commons

\title{
Distribuição do investimento público na assistência fisioterapêutica ambulatorial, Brasil, 2000 a 2006
}

\author{
Distribution of public investments in physical therapy \\ in outpatient care, Brazil, 2000 to 2006
}

\section{Shamyr Sulyvan de Castro ${ }^{[a]}$, Guilherme Rodrigues Barbosa ${ }^{[b]}$, Ricardo Goes de Aguiar ${ }^{[c]}$}

[a] Fisioterapeuta, doutor em Saúde Pública pela Universidade de São Paulo (USP), professor adjunto do Departamento de Fisioterapia Aplicada do Instituto de Ciências da Saúde (ICS) da Universidade Federal do Triângulo Mineiro (UFTM), Uberaba, MG - Brasil, e-mail: shamyr@fisioterapia.uftm.edu.br

[b] Fisioterapeuta, mestre em Saúde Pública pela Universidade de São Paulo (USP), professor assistente da Universidade Federal de Sergipe (UFS), Lagarto, SE - Brasil, e-mail: guirbarbosa@gmail.com

[c] Fisioterapeuta, mestre em Saúde Pública pela Universidade de São Paulo (USP), professor assistente e coordenador do curso de Fisioterapia da Universidade Federal de Sergipe (UFS), Lagarto, SE - Brasil, e-mail: ricardoefisio@hotmail.com

\section{Resumo}

Introdução: 0 estudo da distribuição dos recursos financeiros no âmbito das atividades fisioterapêuticas na esfera do SUS é uma importante ferramenta para gestão dos serviços, assim como dos recursos financeiros, humanos e físicos. Objetivo: Determinar o perfil de distribuição dos recursos destinados à cobertura dos gastos com atendimento em fisioterapia no nível secundário de atenção à saúde, nas regiões do Brasil, de 2000 a 2006. Materiais e métodos: Série histórica com dados a respeito do pagamento de atendimento em fisioterapia no setor secundário nas diversas regiões do país. Os dados foram coletados no site do DATASUS. Foi feita uma análise per capita considerando-se as informações populacionais disponibilizadas pelo IBGE. Efetuou-se análise descritiva e análise de correlação de Pearson. Resultados: A região Nordeste apresentou maior investimento per capita no período estudado, $\mathrm{R} \$ 0,4713$; e a região Norte a menor, $\mathrm{R} \$ 0,2834$. 0 investimento municipal foi o que mais se ampliou em todas as regiões, houve incremento no valor total de recursos empregados no custeio de sessões de fisioterapia, de 2000 a 2006. Conclusões: $O$ investimento de recursos financeiros em fisioterapia no nível secundário de atendimento à saúde mostrou padrões diferentes nas diversas regiões do 
Brasil, com maior contraste entre a região Nordeste e a Norte, com o maior e o menor investimento per capita, respectivamente.

Palavras-chave: Fisioterapia. Economia da saúde. Atenção secundária à saúde.

\section{Abstract}

Introduction: The study of the distribution of financial resources within the physiotherapy activities in the sphere of SUS is an important tool for management services as well as financial, human and physical resources. Objective: To determine the profile of distribution of resources to cover costs of physical therapy sessions in a secondary level of health care in different Brazilian geographic areas. Materials and methods: Time series analysis of physical therapy session payments in the secondary level was accomplished from 2000 to 2006. Data were collected from DATASUS website. Per capita analysis was performed based on population information provided by IBGE. Descriptive analysis and Pearson's correlation were carried out. Results: Northeast region showed the greatest per capita investment in the studied period $(R \$ 0.4713)$, while the North region had the smallest one ( $R \$$ 0.2834). From 2000 to 2006, the city's financial investments in this field increased in all Brazilian regions and the total of resources used to afford physical therapy sessions costs was increased. Conclusions: The investment of resources in physical therapy in a secondary level of health care showed different patterns different Brazilian geographic areas.

Keywords: Physical therapy. Health economics. Secondary health care.

\section{Introdução}

A fisioterapia é definida como "ciência da saúde que estuda, previne e trata os distúrbios cinéticos funcionais intercorrentes em órgãos e sistemas do corpo humano, gerados por alterações genéticas, por traumas e por doenças adquiridas" (1). Essa ciência atua por meio de mecanismos terapêuticos próprios, sistematizados pelos estudos da biologia, das ciências morfológicas, das ciências fisiológicas, das patologias, da bioquímica, da biofísica, da biomecânica, da cinesia, da sinergia funcional, e da patologia de órgãos e sistemas do corpo humano e as disciplinas comportamentais e sociais (1). 0 Coffito define ainda como fisioterapeuta o profissional de saúde com formação acadêmica superior habilitado à construção do diagnóstico dos distúrbios cinéticos funcionais (diagnóstico cinesiológico funcional), a prescrição das condutas fisioterapêuticas, a sua ordenação e indução no paciente e o acompanhamento da evolução do quadro clínico funcional e as condições para alta do serviço (1).

Em uma reflexão epistemológica percebe-se que apesar de a fisioterapia ser uma ciência que estuda, previne e trata os distúrbios cinéticos funcionais, o fisioterapeuta é, sobretudo, um profissional que diagnostica, prescreve e ordena condutas de tratamento e acompanha a evolução do quadro do paciente. Ou seja, enquanto a ciência Fisioterapia pode atuar também na prevenção, o profissional da fisioterapia lida com a doença. Esse e outros fatores, como a grande especialização em métodos e técnicas voltados à reabilitação, contribuíram para que o fisioterapeuta se tornasse um ator fundamentalmente ligado aos dois últimos níveis de atenção à saúde. Entretanto, a inclusão do fisioterapeuta nos diversos níveis de atenção à saúde tem sido objeto de discussão entre profissionais, academia e gestores em saúde $(1,2)$.

Segundo Westphal (3), a organização da atenção em saúde é classicamente dividida em três níveis, originariamente sugerida por Leavell and Clark, a saber: prevenção primária; secundária e terciária. De acordo com esse modelo, a prevenção primária consiste, sobretudo, em cuidados relativos à prevenção das doenças e promoção da saúde. Medidas neste âmbito compreenderiam ações como vacinação (proteção específica ou prevenção das doenças) e também medidas educativas com a finalidade de melhora da resistência e do bem-estar geral (promoção da saúde). A prevenção secundária atuaria nos indivíduos doentes em duas frentes, o diagnóstico e tratamento precoce em pessoas assintomáticas (buscando a diminuição 
da incidência) e a diminuição das incapacidades em pessoas sintomáticas (diminuindo a prevalência). Quando se trata da prevenção terciária, o foco é a reabilitação do paciente.

Assim, a principal função do fisioterapeuta no nível secundário é prevenir ou diminuir o aparecimento de sequelas, atuando na recuperação de pacientes. Com a mudança dos perfis populacional e epidemiológico e o consequente aumento da longevidade e da prevalência de morbidades crônico-degenerativas e de deficiências físicas, o papel do fisioterapeuta tem sido amplamente valorizado $(1,4)$.

0 modelo de atenção à saúde no Brasil tem passado por um processo de profundas alterações, entre um modelo baseado na assistência hospitalar-curativa e um modelo com ênfase na atenção básico-preventiva. Isso pode ser confirmado pelas alterações no fluxo das aplicações financeiras dos gestores de saúde, que, paulatinamente, estão incrementando o repasse de verbas para a atenção básica em detrimento das esferas hospitalares, ou de alta densidade tecnológica (5). Além disso, o sistema de saúde brasileiro tem uma característica importante: a complementaridade pelo sistema privado. Ou seja, empresas privadas têm permissão para prestar serviços em saúde, complementando o sistema público (6). Esse dispositivo legal cria algumas contradições à medida que proporciona um panorama em que as pessoas que procuram um serviço de saúde podem receber dois atendimentos, pelo setor público ou pelo privado, no mesmo estabelecimento de saúde.

0 uso dos recursos humanos e financeiros em todas as áreas da saúde deve ser planejado visando atuar de maneira coerente na prevenção das doenças, bem como no tratamento e na recuperação dos indivíduos já acometidos. 0 conhecimento do perfil da distribuição das verbas que financiam os serviços fisioterapêuticos, aliado à análise do perfil epidemiológico da população, proporciona um melhor planejamento por parte dos gestores, otimizando os gastos e aumentando os benefícios dos serviços de saúde em favor dos usuários do sistema de saúde. Nesse processo, a informação é fundamental para subsidiar a tomada de decisões.

Entre os sistemas de informação em saúde de abrangência nacional, o Sistema de Informação Ambulatorial do Sistema Único de Saúde (SIA-SUS) é o único que registra procedimentos fisioterapêuticos. Criado na década de 1990 com o objetivo de permitir o registro das informações dos atendimentos realizados em regime ambulatorial, especialmente no que se refere à questão contábil, o sistema vem sendo aprimorado no sentido de ser utilizado efetivamente no planejamento, regulação e avaliação da assistência ambulatorial (7). Dessa forma, o SIA-SUS permite o acompanhamento da produção fisioterapêutica ambulatorial no país.

0 presente estudo tem por objetivo determinar o perfil de distribuição dos recursos destinados à cobertura dos gastos com atendimento em fisioterapia no nível secundário de atenção à saúde, também denominado atendimento ambulatorial, nas regiões do Brasil, de 2000 a 2006.

\section{Materiais e métodos}

Estudo descritivo de uma série histórica de dados a respeito do financiamento em saúde de atendimentos em fisioterapia no nível secundário de atenção à saúde, no período de 2000 a 2006.

A presente pesquisa foi realizada a partir da coleta de dados sobre financiamento de atendimento em fisioterapia no nível secundário de assistência à saúde. As informações estão disponíveis no site do DATASUS, por meio do SAI-SUS.

Foram analisadas variáveis referentes ao financiamento de atendimentos de fisioterapia, no nível de serviços especializados, por meio dos repasses feitos pelo governo federal às diferentes esferas de administração dos serviços de saúde. Estudou-se o financiamento nos níveis municipal, estadual e federal e para os serviços privados. Como as diferentes regiões do país apresentam desigualdades no perfil populacional, foi calculado o valor per capita gasto em cada região do país, com base nos dados populacionais e projeções disponibilizados pelo Instituto Brasileiro de Geografia e Estatística (IBGE), segundo a modalidade de administração dos serviços de saúde estudados, para que essas diferenças populacionais não influenciassem as análises.

Os dados foram organizados descritivamente em tabelas e gráficos. Fez-se a análise de correlação de Pearson com a parametrização dos dados, determinada pelo teste de Shapiro-Wilk. Também foi construído um mapa com o gasto per capita total de cada região em todo o período do estudo. Para a análise dos dados, foi utilizado o software Stata 9.2 e para a confecção do mapa, o pacote TabWin. 


\section{Resultados}

No Brasil, no período de 2000 a 2006, houve um repasse total de recursos de quase $\mathrm{R} \$ 123$ bilhões para a Saúde, com média anual de R $\$ 17,5$ bilhões. Essas médias chegaram a R $\$ 7,8$ bilhões no Sudeste; $\mathrm{R} \$ 4,7$ bilhões no Nordeste; $\mathrm{R}$ 2,5 bilhões no Sul; $\mathrm{R} \$$ 1,1 bilhão no Centro-Oeste; e R \$1,1 bilhões no Norte, para o período citado.

Considerando-se o gasto total no país com serviços especializados (nível secundário), no mesmo período, houve um repasse de $\mathrm{R} \$ 25,1$ bilhões, sendo que desse total $1,97 \%$ foi utilizado para o pagamento de atendimentos de fisioterapia; essa porcentagem foi de $1,73 \%$ na região Norte; $2,63 \%$ na região Nordeste; $1,71 \%$ no Sudeste; $2,15 \%$ no Sul; e de $1,55 \%$ no CentroOeste. Os valores brutos investidos no nível secundário, para o período em estudo, foram de $\mathrm{R} \$ 1,6$ bilhão na região Norte; $\mathrm{R} \$ 6,2$ bilhões na região Nordeste; $\mathrm{R} \$ 12,5$ bilhões no Sudeste; $\mathrm{R} \$ 3,0$ bilhões no Sul; e de R $\$ 1,6$ bilhão no Centro-Oeste

0 comportamento dos repasses financeiros do governo federal para o pagamento dos atendimentos em fisioterapia às diferentes esferas de administração dos serviços de saúde demonstra que houve uma tendência de crescimento uniforme dos repasses para o nível municipal em todas as regiões durante o período estudado; apenas na região Sul o aumento não foi tão evidente (Gráfico 1). A crescente municipalização no sistema de saúde brasileiro pode justificar esse evento (7). A proximidade entre o gestor e a população que necessita de assistência fisioterapêutica pode ser o fator sensibilizante para a necessidade de maiores investimentos em serviços de fisioterapia.

Cabe ressaltar a importância do exercício adequado da participação popular e do controle social, princípios constitucionais do SUS, como um mecanismo essencial no estabelecimento das necessidades em saúde, bem como no acompanhamento das estratégias adotadas para lidar com tais demandas.

Com relação aos repasses para o nível estadual, a região Sudeste mostrou valores per capita gradativamente crescentes no período estudado, já a região Norte apresentou decréscimo comparando-se 2001 com 2000, com um discreto aumento nos anos seguintes. A região Nordeste apresentou oscilações até 2004, com elevação posterior. A região Sul mostrou pequena variação no período estudado, havendo tendência à diminuição. Na região Centro-Oeste verificou-se oscilações em torno do valor de $\mathrm{R}$ \$,10 per capita.

Na análise dos dados do nível federal, observaram-se grandes variações que dificultaram o estabelecimento de um padrão, sendo possível afirmar apenas que houve um aumento no período.

As oscilações no repasse per capita para os serviços privados comportaram-se de maneira semelhante em todas as regiões. Entretanto, os valores registrados foram diferentes, ficando em torno de $\mathrm{R} \$$ 0,12 na região Norte; $R \$ 0,30$ na Nordeste; $R \$ 0,18$ na Sudeste; R \$ 0,26 na Sul; e R \$ 0,07 na Centro-Oeste.

A região Nordeste foi a que apresentou a maior média per capita de aplicação de recursos financeiros em atendimentos fisioterapêuticos, seguida em ordem decrescente pelas regiões Sudeste, Sul, Centro-Oeste e Norte (Figura 1).

Analisando os coeficientes de correlação entre o investimento total em atendimentos de fisioterapia e o tempo, verifica-se que houve correlação positiva

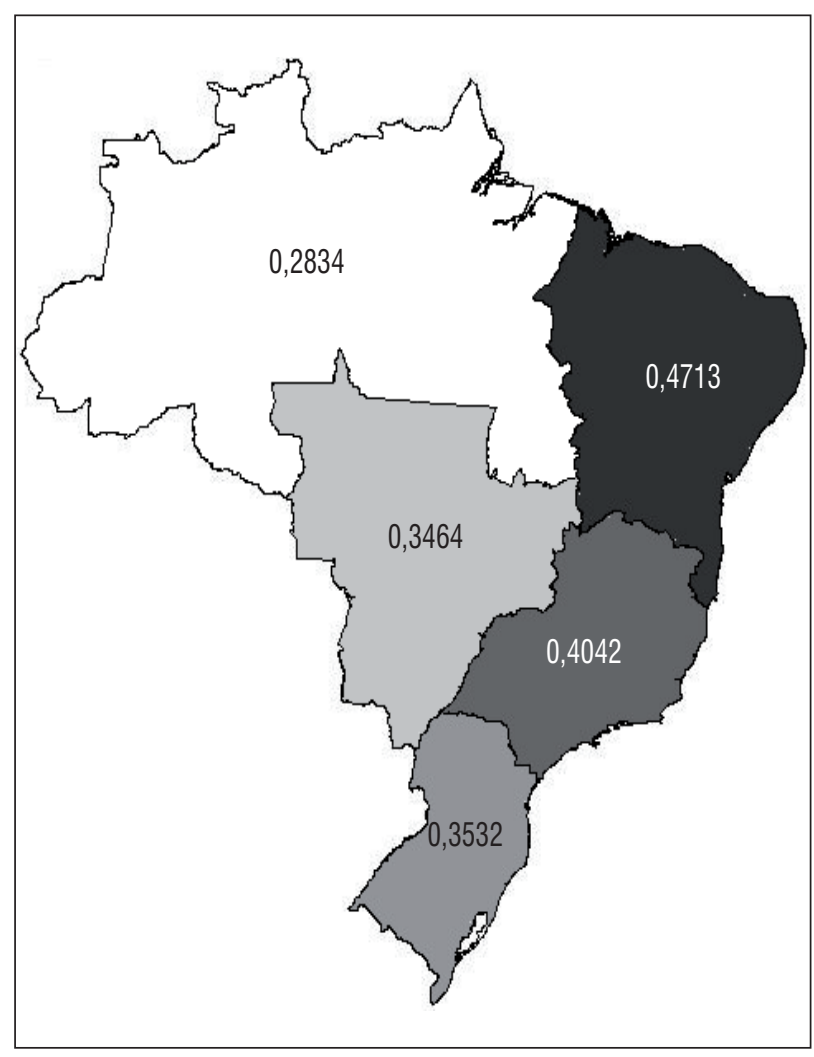

Figura 1 - Distribuição da média per capita de recursos totais investidos em sessões de fisioterapia no nível secundário de atendimento à saúde em cada região do país, no período de 2000 a 2006

Fonte: Dados da pesquisa. 


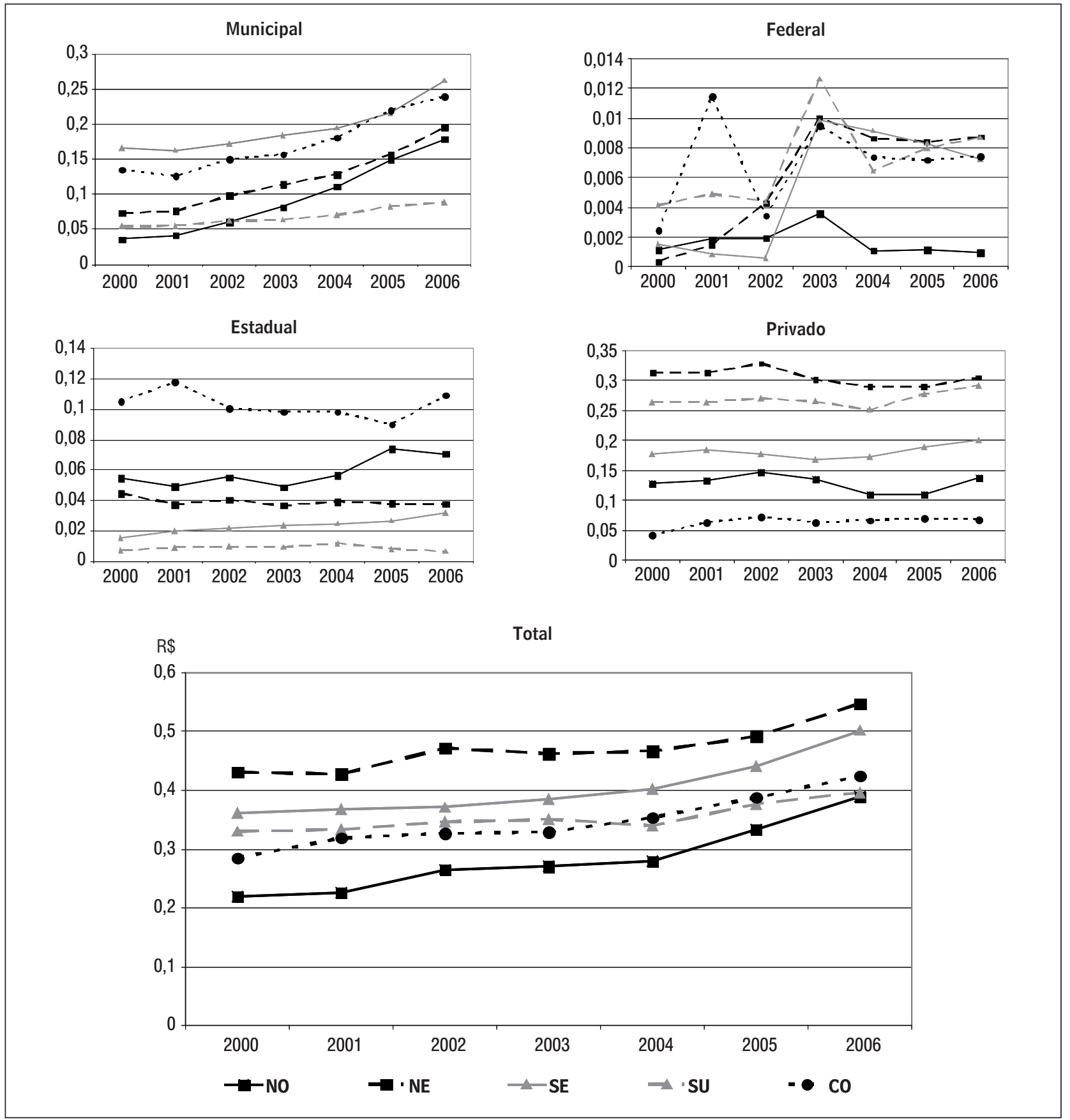

Gráfico 1 - Distribuição do repasse de recursos per capita em diferentes modalidades de gestão e para o total nas regiões do país, 2000 a 2006

Fonte: Dados da pesquisa.

em todas as regiões, quando analisados os dados agregados. Ou seja, com o passar do tempo, houve incremento no repasse de verbas com coeficiente de correlação maior na região Centro-Oeste.

Entretanto, os dados desagregados por modalidade de financiamento mostram que somente no nível municipal houve correlação crescente em todas as regiões. Quando se analisa o nível estadual, somente nas regiões Norte e Sudeste foi encontrada associação estatística significante. Com relação ao nível federal, encontrou-se associação somente na região Nordeste. No setor privado não se encontrou associação estatisticamente significante em nenhuma das regiões do país (Tabela 1 ). 
Tabela 1 - Distribuição dos coeficientes de correlação entre o tempo e o investimento em atendimento em fisioterapia nas diferentes regiões do país, 2000 a 2006

\begin{tabular}{lccccc}
\hline \multirow{2}{*}{ Modalidade de financiamento } & \multicolumn{3}{c}{ Região brasileira } \\
& Norte & Nordeste & Sudeste & Sul & Centro-Oeste \\
\hline Municipal & $0,98^{\mathrm{a}}$ & $0,97^{\mathrm{a}}$ & $0,91^{\mathrm{b}}$ & $0,96^{\mathrm{a}}$ & $0,95^{\mathrm{a}}$ \\
Estadual & $0,76^{\mathrm{c}}$ & $-0,58$ & $0,97^{\mathrm{a}}$ & $-0,04$ & $-0,39$ \\
Federal & $-0,24$ & $0,85^{\mathrm{c}}$ & 0,75 & 0,55 & 0,25 \\
Privado & $-0,27$ & $-0,60$ & 0,52 & 0,55 & 0,65 \\
Total & $0,94^{\mathrm{c}}$ & $0,90^{\mathrm{b}}$ & $0,90^{\mathrm{b}}$ & $0,88^{\mathrm{b}}$ & $0,96^{\mathrm{a}}$ \\
\hline
\end{tabular}

Legenda: $a=p<0,001 ; b=p<0,01 ; c=p<0,05$.

Fonte: Dados da pesquisa.

\section{Discussão}

A região Sudeste se destacou como a que mais recebeu recursos financeiros brutos para o pagamento de atendimento em fisioterapia em um panorama nacional, sendo a região Norte a que apresentou o menor investimento. Esses dados podem ter sua explicação nas diferenças populacionais e econômicas entre as regiões do país.

Quando se analisa o percentual destinado à assistência ambulatorial, observa-se que a região que mais comprometeu seu orçamento com os serviços de fisioterapia em nível ambulatorial foi a região Nordeste, seguida por Sudeste, Sul, Centro-Oeste e Norte, evidenciando que o investimento per capita também obedece a essa sequência de gasto maior no Nordeste e menor no Norte.

Esses dados mostram uma aparente contradição, uma vez que os estados do Nordeste apresentam um perfil epidemiológico diferenciado em relação às regiões Sudeste e Sul. Segundo Villa e Aranda (7), os fatores demográficos e econômicos podem influenciar o estado de saúde das coletividades. Considerando-se que um país com dimensões continentais como o Brasil, que apresenta disparidades econômicas e demográficas regionais, variações na distribuição dos recursos em saúde são esperadas, uma vez que as regiões apresentam diferentes perfis de saúde (9). As variações na mortalidade reforçam esse padrão diferenciado em cada região.

Dados do DATASUS do ano de 2004 ratificam o exposto, fornecendo coeficientes de mortalidade por doenças do aparelho circulatório de 19 a cada mil no Sul; 18,8 no Sudeste; 11,9 no Nordeste; 14,2 no Centro-Oeste; e de 7,2 no Norte. A mortalidade por neoplasias em 2004 também mostra padrões que corroboram a desigualdade na distribuição de eventos em saúde nas diversas regiões do país, com índices de 10,9 a cada mil no Sul; de 9,3 no Sudeste; 6,6 no Centro-Oeste; 3,6 no Norte; e de 4,7 no Nordeste; observa-se que possíveis distorções nos indicadores obtidos na base de dados do DATASUS podem ocorrer, mas que não devem influenciar de forma drástica os coeficientes apresentados anteriormente $(9,10)$.

Cabe ressaltar o problema da definição de causa de óbito, sendo essa uma questão oriunda do preenchimento deficitário das declarações de óbito. E mencionar ainda a existência de desigualdades no uso dos serviços de saúde (11), que são proporcionadas por maior cobertura por planos privados de saúde entre as pessoas com nível socioeconômico mais elevado e também por limitações na capacidade de atendimento da demanda pelos serviços de saúde (13).

As informações anteriores indicam que a região Nordeste dispensa maior atenção a esses serviços de saúde quando comparada às outras regiões. Os reais motivos dessa diferença na distribuição dos recursos financeiros em questão podem estar relacionados a fatores diversos como a cobertura dos planos de saúde e políticas de investimento adotadas pelos gestores. Entretanto, essa análise não faz parte dos objetivos deste estudo, fazendo-se necessários novos estudos.

Em estudo transversal de base populacional realizado na cidade de Pelotas (RS), os autores 
encontraram que a idade esteve diretamente relacionada à utilização de serviços de fisioterapia, ou seja, usuários de idade mais avançada utilizaram mais esses serviços. 0 estudo salienta ainda a atuação fisioterapêutica nas condições crônico-degenerativas. Dos indivíduos pesquisados, $66 \%$ utilizaram serviço público de fisioterapia, enquanto $25 \%$ serviram-se de convênios e apenas $9 \%$ fizeram uso de serviço privado (13). Esses dados evidenciam não só uma maior necessidade de serviços de fisioterapia por populações com idade avançada, mas também o maior uso dos serviços públicos de saúde.

\section{Considerações finais}

Os investimentos, em sua totalidade, mostraram tendência mais clara ao aumento ao longo do tempo, quando comparadas as esferas separadamente. No entanto, essa tendência para todas as regiões foi mais perceptível no nível municipal, que foi o principal gestor dos serviços de fisioterapia no atendimento secundário, juntamente com o setor privado. Novos estudos devem ser realizados para que esse padrão seja confirmado e explicado.

\section{Referências}

1. Conselho Federal de Fisioterapia e Terapia Ocupacional - COFFITO. Fisioterapia - definição. 2010 [acesso 23 abr 2010]. Disponível em: http://www.coffito.org. $\mathrm{br} /$ conteudo/con_view.asp?secao $=27$

2. Castro SS, Cipriano G Junior, Martinho A. Fisioterapia no Programa de Saúde da Família: uma revisão e discussões sobre a inclusão. Fisioter Mov. 2006;19(4):55-62.

3. Westphal MF. Promoção da saúde e prevenção de doenças. In: Campos GWS, Minayo MCS, Akerman M, Drumond M Junior, Carvalho YM. Tratado de saúde coletiva. São Paulo: HUCITEC; 2006. p. 635-668.

4. Carvalho DMT. Financiamento da assistência médico-hospitalar no Brasil. Ciênc Saúde Coletiva. 2007;12(4): 879-92.

5. Santos IS, Ugá MAD, Porto SM. O mix público-privado no sistema de saúde brasileiro: financiamento, oferta e utilização de serviços de saúde. Ciênc Saúde Coletiva. 2008;13(5):1431-40.
6. Brasil. Ministério da Saúde. Secretaria de Atenção à Saúde. Sistema de Informações Ambulatoriais do SUS SAI/SUS: manual de orientações técnicas. Brasília: Editora do Ministério da Saúde; 2007.

7. Villa VM; Aranda MP. The demographic, economic, and health profile of older Latinos: implications for health and long-term care policy and the Latino family. J Health Hum Serv Adm. 2000;23(2):161-80.

8. Lima LD, Andrade CLT. Condições de financiamento em saúde nos grandes municípios do Brasil. Cad Saúde Pública. 2009;25(10):2237-48.

9. Brasil. Ministério da Saúde. Departamento de Informática do SUS - DATASUS. Indicadores de mortalidade - taxa de mortalidade específica por doenças do aparelho circulatório. 2006 [acesso 30 abr 2010]. Disponível em: http://tabnet.datasus.gov.br/cgi/ tabcgi.exe?idb2006/c08.def

10. Brasil. Ministério da Saúde. Departamento de Informática do SUS - DATASUS. Indicadores de mortalidade - Taxa de mortalidade específica por neoplasias malignas. 2006 [acesso 30 abr 2010]. Disponível em: http://tabnet.datasus.gov.br/cgi/tabcgi. exe?idb2006/c10.def

11. Szwarcwald CL, Souza PR Júnior, Damacena GN. Socioeconomic inequalities in the use of outpatient services in Brazil according to health care need: evidence from the World Health Survey. BMC Health Serv Res. 2010;10:217. doi: 10.1186/1472-6963-10-217

12. Siqueira FV, Facchini LA, Hallal PC. Epidemiology of physiotherapy utilization among adults and elderly. Rev Saúde Pública. 2005;39(4):663-8.

13. Ugá MA, Santos IS. An analysis of equity in Brazilian health system financing. Health Aff (Millwood). 2007;26(4):1017-28.

Recebido: 03/12/2012

Received: $12 / 03 / 2012$

Aprovado: 20/06/2013

Approved: 06/20/2013 